\section{Up-shoot in adduction has no prognostic value for decompensation in refractive accommodative esotropia}

Abstract

Purpose To assess whether up-shoot (elevation) in adduction has any prognostic value for the functional outcome of binocular vision in fully refractive accommodative esotropia (RAET).

Methods A retrospective study was performed on 58 patients initially diagnosed with RAET who were followed for at least 4 years. Results Without glasses, up-shoot in adduction with or without a $\mathrm{V}$ pattern was detected in 22 of 51 patients who maintained normal binocular alignment during the whole follow-up and in 3 of 7 patients who lost normal binocular vision and showed a manifest esotropia despite glasses at any time of the follow-up. Up-shoot in adduction was still present through glasses only in 3 patients among these 25 cases. Two of these remained fully compensated with glasses during the considered period. The third case developed up-shoot in adduction after decompensation of a previously compensated strabismus. Conclusions Up-shoot in adduction is not a common sign in RAET. When present, it does not necessarily represent a negative prognostic sign for preservation of normal binocular vision through glasses in this condition. Upshoot in adduction can develop after the loss of alignment in some decompensated patients. There is therefore no evidence in favour of a cause-effect relationship between up-shoot in adduction and loss of binocularity in RAET.

Key words Binocular vision, Inferior oblique muscle overaction, Refractive accommodative esotropia, Strabismus surgery, Up-shoot in adduction, $\mathrm{V}$ pattern

Overaction of the inferior oblique muscle with up-shoot (elevation) in adduction may be secondary to paresis or paralysis either of the ipsilateral superior oblique muscle or of the contralateral superior rectus muscle.
Nevertheless, up-shoot in adduction may be also encountered in subjects in whom no other form of strabismus is present or in cases where there is no evidence for a past or present ipsilateral superior oblique paralysis. In fact, up-shoot in adduction typically occurs in essential infantile esotropia, ${ }^{1}$ where it is commonly considered to be caused by a 'primary' inferior oblique overaction. At present, no convincing explanations have been put forward regarding the existence of a true primary inferior oblique muscle overaction, mostly in essential infantile esotropia. Other causes for up-shoot in adduction in the absence of a deficit of superior oblique or superior rectus muscles have been suspected. In essential infantile esotropia a dissociated vertical deviation (DVD) may cause the adducted eye which is covered by the nose to turn upward. ${ }^{2}$ In Duane syndrome, elevation in adduction is caused by a bridle effect of a co-contracted lateral rectus muscle or by a pathological cofiring of the medial rectus and the superior rectus muscles. ${ }^{3}$ Recently an inferior displacement of the pulley of the lateral rectus muscle has been observed with high-resolution magnetic resonance imaging in patients who showed up-shoot in adduction of the nonfixating eye. ${ }^{4}$ From a clinical point of view, upshoot in adduction in convergent strabismus is a finding commonly considered as an indirect sign of early-onset strabismus without normal binocular vision.

Refractive accommodative esotropia (RAET) is generally managed by fully correcting the hypermetropic refractive error. However, unexplainably surgery is advocated in this condition in order either to eliminate glasses or to correct any supposed dysfunction of the oblique muscles, mostly overaction of the inferior oblique muscles, which is thought to induce loss of binocular alignment. ${ }^{5}$

Advocates of surgery for fully RAET base their indications on a supposed primary dysfunction of the oblique muscles, which
C. Schiavi

C. Bellusci

E.C. Campos

Eye Service

University of Bologna

School of Medicine

Bologna, Italy

Prof. Emilio C. Campos Clinica Oculistica dell'Università via Massarenti 9 I-40138 Bologna, Italy

Tel: +39 (0)51341450

Fax: +39 (0)51 342516

e-mail:

campos@alma.unibo.it

Received: 17 March 2000 Accepted in revised form: 27 July 2000 
Table 1. Materials

\begin{tabular}{lr}
\hline No. of RAET patients & 58 \\
Age at first examination (months) & $26( \pm 8)$ \\
Refractive error (at first examination) & $+4.5( \pm 1)$ \\
Follow-up (years) & $7.5( \pm 2)$ \\
\hline
\end{tabular}

Values are mean $( \pm \mathrm{SD})$.

hampers fusion in this condition. They therefore perform a simultaneous horizontal and cyclovertical surgery in order not only to eliminate glasses, but also to prevent a possible deterioration of binocular function..$^{5-8}$ Such an assumption is generally rejected because the theory behind it is not supported by convincing scientific evidence. ${ }^{9}$ In fact, reduced or even absent stereopsis is not an uncommon observation in patients with RAET, but it could be explained as a consequence of disruption of binocular vision during visual immaturity, prior to correction of the esotropia with glasses. ${ }^{10}$

Clinical observations may suggest that up-shoot in adduction can be observed in patients with RAET who decompensate, either because of inadequate optical correction, or even in spite of the full optical correction of the hypermetropic refractive error. In order to assess whether a cause-effect relationship exists between the presence of up-shoot in adduction and risk of disruption of normal binocular vision in RAET, we performed a retrospective study on a series of patients initially diagnosed with RAET, followed for at least a 4 year period.

\section{Materials and methods}

We reviewed the files of 58 patients initially diagnosed with RAET (Table 1) who have been followed for at least a 4 year period, with an average follow-up of 7 years and 6 months (range 4-12 years). All the patients included in this study showed a manifest esodeviation at near and distance fixation prior to correction of the hypermetropic refractive error. These patients were selected on the basis of the presence of normal binocular vision, i.e. normal sensory and motor fusion and stereopsis, orthophoria or mild esophoria for near and distance fixation with the full retinoscopic correction of their hypermetropia at least in the beginning of the observation. At the first examination, the age of the patients ranged from 14 months to 4 years and 3 months (mean age 2 years and 2 months). None of these patients showed anisometropia and refractive error (spherical equivalent) determined at the first examination ranged from +3 dioptres (D) to +7 $\mathrm{D}$ (mean $+4.5 \mathrm{D})$.

The deviation was measured yearly with the prism cover-test at near $(33 \mathrm{~cm})$ and distance $(5 \mathrm{~m})$ fixation, first without glasses and then with the full retinoscopic correction of the hypermetropic error. Accommodative targets were used for measurements at near distance. The 4 prism dioptre base-out prism test (Irvine test) was used to check the presence of normal fusional vergences.

Stereopsis was tested with glasses using the random-dot TNO test or the Lang test. Records of ocular motility examination for each patient during the whole follow-up
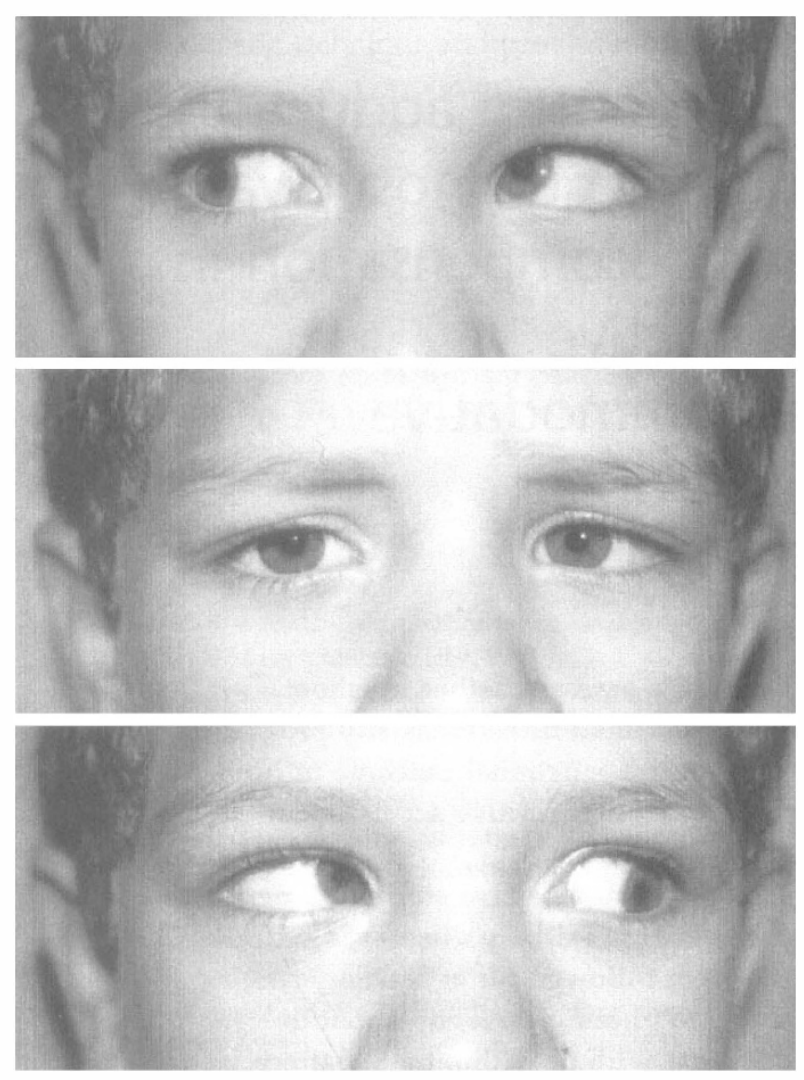

Fig. 1. An apparent up-shoot in adduction is observed when testing a patient with RAET without his spectacles. Note the manifest esodeviation of the left eye.

period were evaluated in order to assess the prevalence of dysfunction of the oblique muscles and of alphabetical patterns, both in those patients who maintained normal binocular vision and in those who showed a deterioration of binocular function during the observation time. A and V patterns were defined when the difference of the horizontal deviation between upward and downward gaze was 15 prism dioptres or more. Up-shoot in adduction was assessed clinically, i.e. by examining ductions and versions, both through the optical correction and without glasses. In our records, it was graded on a clinical scale ranging from +1 to +3 .

\section{Results}

Fifty-one of 58 patients maintained normal binocular function, i.e. stable sensory and motor fusion and stereopsis with glasses, during the whole follow-up period. Minimal $(+1)$ to moderate $(+2)$ up-shoot in adduction was reported in 22 of these 51 fully compensated patients when tested without the optical correction (Fig. 1) and in the presence of a large-angle manifest esodeviation. Five of these 22 patients also exhibited a $\mathrm{V}$ pattern when the deviation was measured with the prism cover-test without glasses at near fixation. No evident deficit of the superior oblique muscles and of the superior rectus muscles was observed in these cases. Up-shoot in adduction disappeared in all but 2 of these 22 patients when ocular motility was examined with the full retinoscopic correction of the hypermetropic 

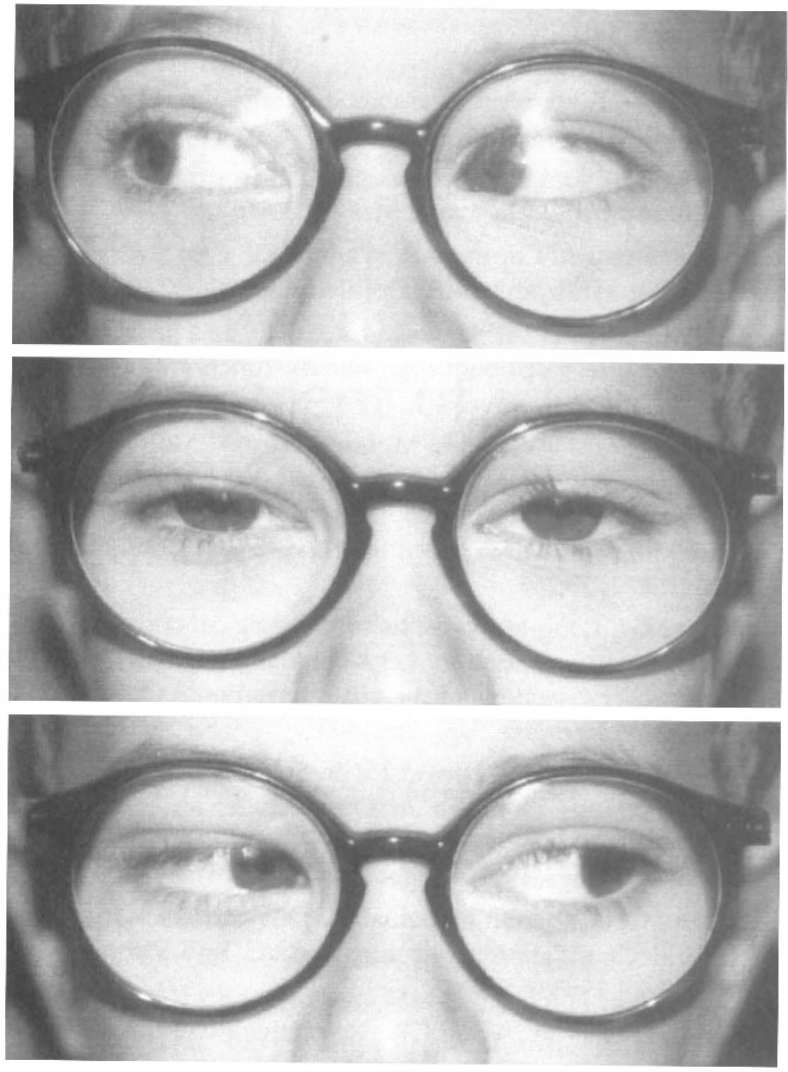

Fig. 2. The same patient as in Fig. 1 with his positive glasses. The upshoot in adduction is no longer evident when the eyes are viewed straight through glasses.

refractive error (Fig. 2). Moreover, no alphabetical pattern was observed through glasses in these 22 patients except for the 2 cases who showed up-shoot in adduction even when wearing the optical correction. In these two cases, a V pattern was found with compensated exophoria in up-gaze and compensated esophoria in down-gaze when tested with glasses.

The remaining 7 patients of our study showed a progressive decompensation of the deviation at some stage of the observation period which had required surgery. After recession of both medial rectus muscles, 5 of them regained motor and sensory fusion and stereopsis with glasses, whereas the remaining 2 patients lost normal binocular vision and stereopsis. These two patients developed a partially accommodative esotropia with a small-angle basic esodeviation at near and distance fixation through glasses despite surgery. Before surgery, a minimal $(+1)$ to moderate $(+2)$ up-shoot in adduction had been observed in 2 of the 5 patients who afterwards regained fusion after a recession of both medial rectus muscles and in 1 of the 2 patients whose normal binocular vision could not be restored with a bimedial recession (Table 2). This mild up-shoot in adduction was observed only when the patients were tested without glasses. After surgery, the up-shoot in adduction without glasses was unmodified in the 2 patients who regained stable motor and sensory fusion, while it worsened into a +3 up-shoot which was evident even with glasses in the patient who developed a
Table 2. Up-shoot in adduction in 58 RAET patients

Evidence of up-shoot in adduction in 51 compensated RAET patients $22 / 51$ patients without hypermetropic correction

$2 / 22$ patients with hypermetropic correction

Evidence of up-shoot in adduction in 7 decompensated RAET patients $3 / 7$ patients without hypermetropic correction

$1 / 3$ patients with hypermetropic correction

partially accommodative esotropia with loss of normal binocular vision. No alphabetical pattern had been reported in the other 6 patients who required surgery.

Finally there was no evidence of any signs of downshoot (depression in adduction) in any of our 58 patients either without glasses or through the optical correction.

\section{Discussion}

Our results confirm previous observations which exclude the possibility that true up-shoot in adduction as well as true $\mathrm{V}$ patterns or other cyclovertical dysfunctions of ocular motility are common findings in RAET. ${ }^{9}$ These supposed vertical incomitancies are detectable only when testing patients without their spectacles in the presence of a large angle manifest esodeviation. Furthermore, when present they do not necessarily represent a negative prognostic sign for preservation of normal binocular vision in this condition. This is in contrast to what is stated by proponents of desagittalisation procedures on the oblique muscles in addition to horizontal surgery in RAET. ${ }^{5-8}$

Moreover a recent study has provided evidence that what appears as primary overaction of an oblique muscle may be a secondary overaction of both elevators. In the presence of inferior displacement of lateral rectus muscle pulleys, the ipsilateral elevators need to contract to maintain a constant vertical eye position. According to Hering's law the contralateral elevators will receive equal innervation, elevating the eye as it is adducting. ${ }^{4}$

The origin of up-shoot in adduction and of the $\mathrm{V}$ pattern which can sometimes be observed when testing patients with fully compensated RAET without optical correction is not easily explainable. Some speculations seem appropriate. While testing patients without correction of their hypermetropia, the accommodative effort causes one eye to deviate. When the eyes are turned in lateral gaze, the nose may act as a further dissociating factor. Therefore, it can be hypothesised that temporary loss of alignment, or the interruption of motor fusion, may cause the adducted eye to turn upwards.

It has been found that even in healthy volunteers with normal stereovision, patching one eye for 3 days can cause up-shoot and down-shoot in adduction. ${ }^{11}$ It is possible, therefore, that up-shoot in adduction reflects latent motility disorders that become manifest by disruption of fusion.

Elevation in adduction which takes place when the eyes are dissociated could also be interpreted as a dissociated vertical deviation (DVD). ${ }^{2}$ Another explanation is that up-shoots in adduction and alphabetical patterns can be attributable to fluctuations 
of accommodation in lateral, upward and downward gaze. As a consequence, these vertical incomitancies are only apparent and must be considered as artefacts without any clinical significance. ${ }^{7}$ In any case there is no convincing explanation as to why only a few patients with RAET show up-shoot in adduction when evaluating ductions and versions without spectacles. It is likely that in some patients with RAET who decompensate, a true up-shoot in adduction can develop after the loss of alignment with a clinical pattern similar to what is commonly observed in essential infantile esotropia. At present we are unable to explain the aetiology of this upshoot in adduction which sometimes can be found in decompensated RAET. It can only be supposed that the loss of alignment, or the deterioration of binocular functions, or both, are somehow involved in inducing up-shoot in adduction. Whether elevation in adduction in these cases should be attributed to overaction of the inferior oblique muscles, or to a DVD, is not easy to establish. ${ }^{2}$ It would be of great interest to know whether a critical time exists within which the deterioration of binocular functions is able to cause up-shoot in adduction to develop. Finally, up-shoot in adduction is often evident in patients with acquired esotropia who regain normal binocular vision after surgical correction. Therefore the evidence of up-shoot in adduction in esotropic patients is not necessarily a sign of congenital origin of strabismus.

\section{References}

1. Noorden GK von. A reassessment of infantile esotropia. Am J Ophthalmol 1988;105:1.

2. Spielman A. Les strabismes: de l'analyse clinique à la synthèse chirugicale. Paris: Masson 1990.

3. DeRespinis PA, Caputo AR, Wagner RS, Guo S. Duane's retraction syndrome. Surv Ophthalmol 1996;40:423.

4. Clark RA, Miller JM, Rosenbaum AL, Demer JL. Heterotopic muscle pulleys or oblique muscle dysfunction? J AAPOS 1998;2:17.

5. Fells P, Bérard PV, Gobin M, Jampolsky A, Molteno A, Noorden GK von, et al. Symposium on the management of fully accommodative esotropia. In: Campos EC, editor. Proceedings of the 6th Meeting of the ISA, Surfer's Paradise, Australia, 1990:269.

6. Gobin MH. Long-term results of the surgical correction of accommodative squint. In: Lenk-Schafer M, editor. Orthoptic horizons. Transactions of the 6th International Orthoptic Congress, Harrogate, UK, 1987:403.

7. Bérard PV, Reydy R, Bérard PV Jr. Surgery of accommodative esotropia: In: Lenk-Schafer $M$, editor. Orthoptic horizons. Transactions of the 6th International Orthoptic Congress, Harrogate, UK, 1987:396.

8. Gobin $\mathrm{MH}$. La vision binoculaire après correction chirurgicale du strabisme accommodatif. Bull Mem Soc Fr Ophtalmol 1985;96:95.

9. Noorden GK von, Avilla CW. Refractive accommodative esotropia: a surgical problem? Int Ophthalmol 1992;16:45-8.

10. Noorden GK von, Avilla CW. Accommodative convergence in hypermetropia. Am J Ophthalmol 1990;110:287-92.

11. Liesch J, Simons HJ. Up- and downshoot in adduction after monocular patching in normal volunteers. Strabismus 1993;1:25-36. 